\title{
Signal hole repair strategy based on sensor deployment density for mobile crowd network
}

\author{
Yong-qiang He
}

\begin{abstract}
In order to reduce the signal holes in wireless sensor networks, we proposed a sensor deployment density-aware signal hole repair strategy of mobile crowd network. On the one hand, based on the multi-dimensional connected graph, the density continuous jitter problem of the sensor network is solved effectively. Sensor density prediction method improves the efficiency of random deployment of sensor nodes. Based on the above schemes, the sensor deployment density sensing model is proposed. On the other hand, according to the regional geometry, diversity of the sensor network channel and density can detect the time domain channel impulse response signal successfully. Finally, the network signal is detected by the signal detection of the mobile crowd network. The signal holes can be detected and repaired based on coverage density and crowd. Experimental results show that the proposed algorithm has outstanding performance in terms of signal strength and signal void ratio compared with the energy-aware repair algorithm.
\end{abstract}

Keywords: Wireless networks, Sensor deployment density, Mobile crowd signal, Repair signal hole

\section{Introduction}

Random deployment of wireless sensor networks [1] is used to easily generate coverage holes. A plurality of wireless sensor networks which are not connected with each other [2] causes the upper layer network to be unable to transmit the damaged network signals correctly. At the same time, it brings a series of signal holes [3]. However, there is difficulty in repairing the network coverage hole [4] and the signal cavity through the use of wireless sensor network in a timely manner. These problems lead to a sharp decline in the performance of wireless sensor networks. Therefore, how to detect the coverage hole [5] and the signal hole becomes the key, how to repair the wireless sensor network's signal cavity becomes the question which needs to be solved urgently.

The following research results show the signal detection schemes. The traditional receiver operating characteristic analysis was extended for single-signal detection and classification of multiple signals [6]. The authors presented the comprehensive analysis of strength-based optimum

Correspondence: heyongqiangxz@163.com

School of Computer, Henan University of Engineering, Zhengzhou, Henan 451191, China

(c) The Author(s). 2017 Open Access This article is distributed under the terms of the Creative Commons Attribution 4.0 International License (http://creativecommons.org/licenses/by/4.0/), which permits unrestricted use, distribution, and reproduction in any medium, provided you give appropriate credit to the original author(s) and the source, provide a link to the Creative Commons license, and indicate if changes were made. signal detection model for concentration-encoded molecular communication with spike transmission based on amplitude-shift keying and on-off keying modulations [7]. The effect of varying the antenna spacing on the received signal correlation was investigated and its subsequent effect on the detection performance was shown by Bhatti et al. [8]. Under a Neyman-Pearson hypothesis-testing problem model, Lei et al. [9] proposed a new detection scheme referred to as the likelihood ratio test with sparse estimation. Based on this property, Gao et al. [10] proposed a low-complexity signal detection algorithm based on the successive over-relaxation method to reduce the overall complexity by one order of magnitude with a negligible performance loss.

In the network signal repair, there are a lot of research results. Based on signal-to-interference-plus-noise ratio (SINR) matrix, Naresh et al. studied the vertical handoff combined with local route repair to improve performance of 4G-Multiradio Mesh Network [11]. Cadambe et al. solved the problem of repair bandwidth minimization by adopting into the distributed storage context an asymptotically optimal interference alignment scheme for large wireless interference networks [12].

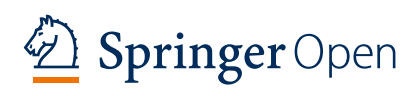


After the deep analysis of receiver operating characteristic analysis [6], received signal correlation [8], and bandwidth minimization [12], we study the sensor deployment density of mobile crowd network and proposed the signal hole repair strategy.

The rest of the paper is organized as follows. Section 2 describes the sensor deployment density sensing model. Section 3 studies the signal detection scheme for mobile crowd network. The signal hole repair strategy for mobile crowd network is shown in Section 4. The performance analysis of power control mechanism has been shown in Section 5. Finally, the conclusions are given in Section 6.

\section{Sensing model with deployment density}

According to the sensing direction and the sensor deployment requirements, we proposed the sensor density sensing model. The problem of exposure path and hidden path of sensor is analyzed by using the multidirection connected graph. Analysis of density continuous jitter in sensor networks is completed by using multi-dimensional connected graph. The sensor nodes are randomly deployed by using the sensor density prediction method. In the dynamic multi-dimensional sensor networks, the density function of the sensor is similar to the K-dimensional Poisson distribution. The weight of this distribution is the sensor position intensity $L_{p}$. In polygon area, the sensor node density $\operatorname{DES}(F)$ is equal to $D_{F}$, which follows the Poisson distribution. $\left\|S_{p}\right\|$ is a polygon area. As a result, the probability density function of sensor deployment is

$$
\left\{\begin{array}{l}
p\left(\operatorname{DES}(F)=D_{F}\right)=\frac{\sqrt{\left(\left\|S_{p}\right\| L_{p}\right)}}{k} \log \left(-k\left\|S_{p}\right\|\right) \\
L_{p}=\exp \left(\frac{-k}{\left\|S_{p}\right\|}\right)
\end{array}\right.
$$

The number of randomly deployed sensor nodes in the direction is determined by the area and the area density. Data forwarding in wireless sensor networks exhibits a time linear and spatial nonlinearity. When the sensor node of a certain density fails, the sensor node data transmission vector of the density trend is shown in Fig. 1. There are some forwarding vectors and density trend sensors in Fig. 1. The density trend sensors can analyze and give the density trend of sensors and vectors. For randomly deployed sensor networks, the energy utilization of a certain direction can be calculated by the data forwarding vector, as shown in formula (2).

$$
\operatorname{EF}\left(S_{0}\right)=L_{p} \int_{i=1}^{k} \operatorname{DES}\left(S_{i}\right) \sum_{i=1}^{n}\left\|D_{i}\right\|^{2}
$$

Here, EF $\left(S_{0}\right)$ represents the sensor network data transmission from the starting point $S_{0}$ in Fig. 1. Parameter $n$ represents the number of sensor nodes in the direction. $D_{i}$ represents the data amount sent by the sensor node.

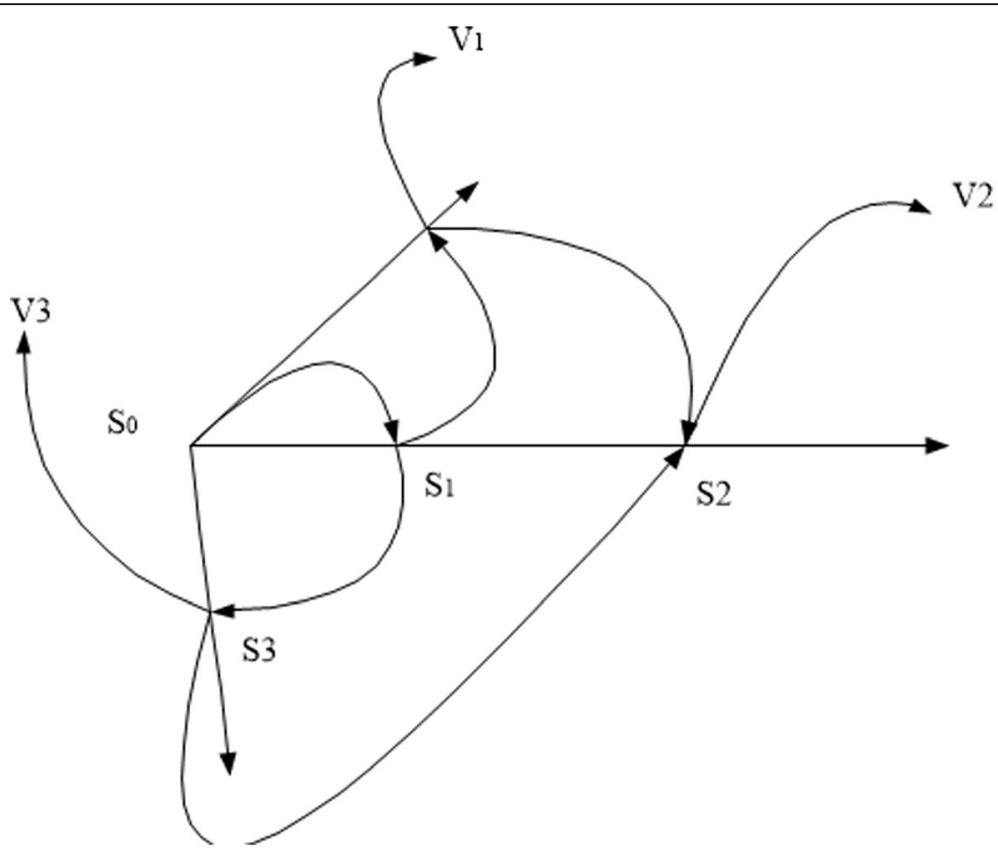

Fig. 1 Data forwarding vector of density trend sensor node 
The relationship between the sensor deployment density sensing vector and the regional area is shown in formula (3).

$$
g=\frac{\operatorname{DES}(F)}{\left\|S_{p}\right\|}\left(1-e^{-L_{p}}\right)
$$

The parameter $g$ represents the ratio of the density in the area. By analyzing the ratio, the sensor deployment can be accurately evaluated by the density coverage performance.

\section{Signal detection scheme of mobile crowd network}

Because of a variety of wireless environment reflector, the directional signal intensity of the sensor is easy to be weakened. Due to the abnormal linear resolution of the signal bandwidth in the environment, the short distance signal of the sensor node is easy to be distorted. It is difficult to detect the high difficulty degree of the linear resolution network signal in multi-path transmission, as shown in Fig. 2.

For the sensor network channel model of Fig. 2, the expression of the time domain channel impulse response signal detection is presented based on the geometry and density of the region, as shown in formula (4).

$$
\left\{\begin{array}{l}
h(t, \varphi)=D_{t} \varphi L_{p}{ }^{\varepsilon}, d<d_{\mathrm{TH}} \\
h(t, \varphi)=\sum_{i=1}^{n} D_{t}(n) \prod_{t} L_{p}{ }^{\varphi}, d \geq d_{\mathrm{TH}}
\end{array}\right.
$$

Here, $h(t, \varphi)$ is the signal detection vector. Time domain channel impulse response delay is $\varphi$ TH denotes the distance threshold. The detection vectors of short distance and long distance are given respectively.
Mobile crowd network signal detection strength is expressed in formula (5).

$$
y=\sum_{i=1}^{L} L_{p}\left(h_{i}\right)+\sum_{j=1}^{M} h_{j}^{k}
$$

Here, symbol $L$ is the length of crowd signal memory which is multi-path transmission. This parameter is used to determine the strength of crowd signal with a different density. $M$ represents the information symbol length of the mobile crowd transmission data signal.

$U_{C}=\left[0, s_{1}, \ldots, s_{x}\right]^{T}$ is the crowd state jitter vector of the mobile network signal detection. There are three kinds of jitter vectors: 0,1 , and 2 . Here, number 0 denotes that the node is static. Number 1 expresses that there is not crowd jitter. Number 2 denotes the signal detection of crowd network jitter. Therefore, the state transition matrix of the mobile crowd network signal detection is as follows:

$$
F=\left[\begin{array}{llll}
0 & 1 & 2 & 0 \\
1 & 0 & 2 & 1 \\
2 & 1 & 0 & 0 \\
1 & 2 & 1 & 0
\end{array}\right]
$$

\section{Signal hole repair strategy for mobile crowd network}

Symbol $G$ represents the density of the mobile crowd network. Symbol $B$ represents the density of the covered area. $R_{G}$ represents the maximum radius of the rule coverage area. The sensing radius of the sensor node is $R_{S}$. Mobile crowd network signal empty vector is $C_{S}$.

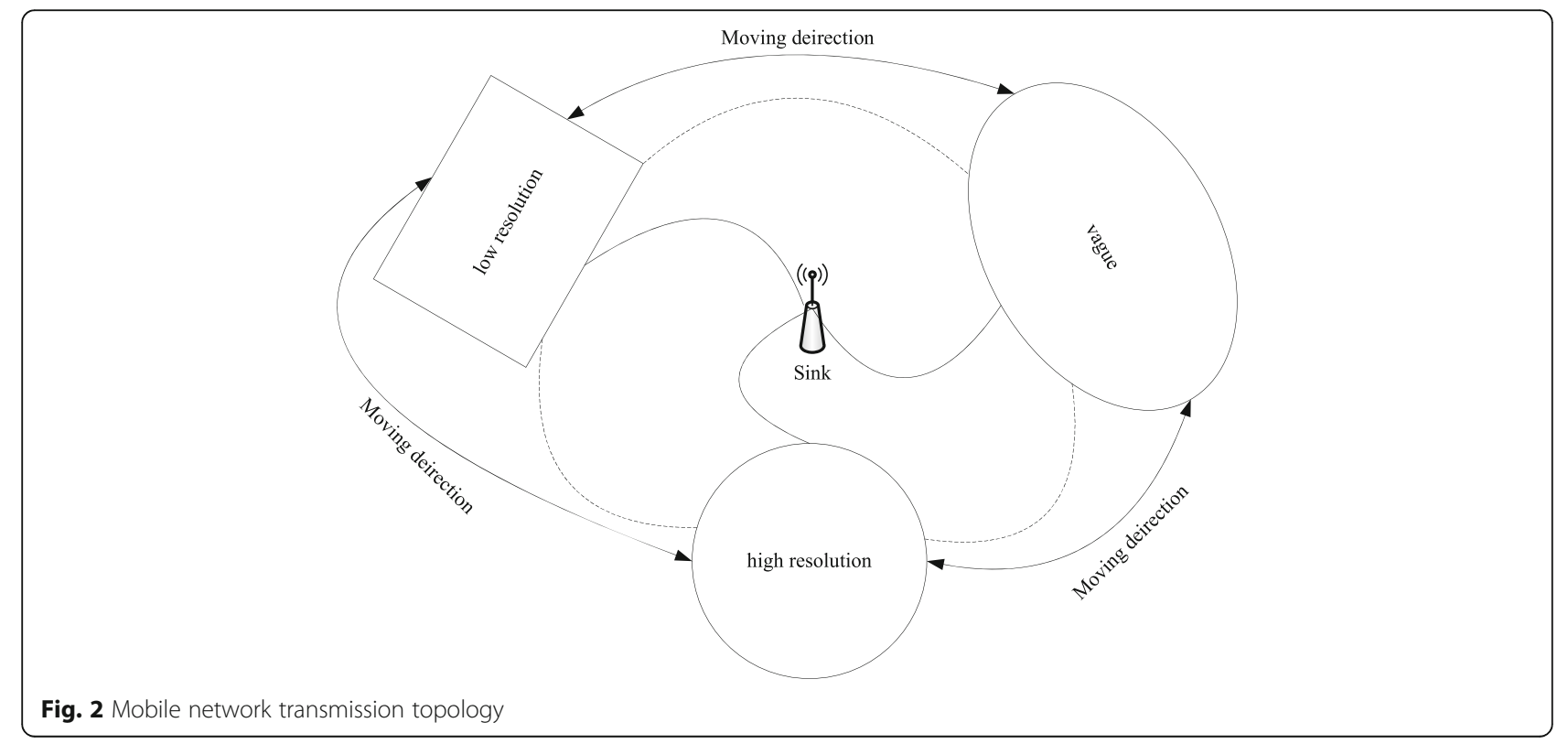


The core idea of mobile crowd network signal hole repair strategy is as follows: Firstly, the coverage area and density of the sensor nodes are obtained. Then, by using the ratio of the Poisson distribution and the area density, the density coverage performance of the sensor deployment is accurately evaluated. Then, through the analysis of the linear resolution and the signal detection of the mobile crowd network, the signal holes would be found. Finally, the connectivity graph of the mobile crowd network is erotic, and the hole is repaired with the fusion degree of the crowd signal.

The specific implementation steps are as follows:

The first step: the sensor nodes would form a Kdimensional connectivity map, which should satisfy the deployment density requirements.

The second step: based on the combination of the linear time resolution characteristics, the sensor network with the orientation, direction, and custom direction would be reconstructed.

The third step: the minimum node number of the coverage requirement is calculated by using the density of the covered area and the maximum radius of the rules:

$$
N_{G}=\left\lfloor B \frac{R_{G}}{\varphi L_{p}}\right\rfloor
$$

The fourth step: in order to establish the network, the sensor nodes should be distributed as evenly as possible in each signal hole through the introduction of crowd network signal detection. Nodes with high degree of integration are distributed in the mobile crowd signal holes. In order to fill the void of the cover signal, nodes with a high degree of convergence and the crowd distribution are used.

The fifth step: in order to reduce the correlation degree between the network signal holes, state transition of the crowd network nodes would be completed according to the status vector.

The signal hole would be repaired and sensor nodes would be active by using the similar computation of Poisson distribution. At the same time, the probability density of the position of these nodes is calculated. Based on the sensing radius of the sensor nodes and the empty vector of the crowd network, the probability density is optimized. This can increase the sensor network coverage and sensor density sensing accuracy.

The sixth step: through the mobile crowd network signal detection, the network signal hole would be found and located.

Algorithm is described as follows:
Algorithm: mobile crowd network signal hole repair strategy denoted as HR-DAC

Input: $G, B, R_{G}, R_{S}, C_{S}, d$

Output: DES, $L_{p}, h(t, \varphi), F($ hole)

1 initialize $D_{F}, k$;

2 measure the $\mathrm{Sp}$;

$3 L_{p}=\exp \left(\frac{-k}{\left\|S_{p}\right\|}\right)$;

$4 p\left(\operatorname{DES}(F)=D_{F}\right)=\frac{\sqrt{\left(\left\|S_{p}\right\| L_{p}\right)}}{k} \log \left(-k\left\|S_{p}\right\|\right)$;

5 measure $S_{0}$;

6 compute $\mathrm{EF}\left(S_{0}\right)$ based on density trend;

7 compute $g$ for evaluating the network coverage ratio;

8 Network signal detection based on region shape;

9 IF $d<d_{\mathrm{TH}}$, go to 10; else go to 11;

$10 h(t, \varphi)=D_{t} \phi L_{p}^{\varepsilon}$;

$11 h(t, \varphi)=\sum_{i=1}^{n} D_{t}(n) \prod_{t} L_{p}{ }^{\varphi}$;

$12 y=\sum_{i=1}^{L} L_{p}\left(h_{i}\right)+\sum_{j=1}^{M} h_{j}^{k}$

13 Finding signal holes;

14 repair signal hole with $N_{G}$.

\section{Analysis of filter algorithm}

We contrast compared the proposed HR-DAC with the energy aware signal restoration algorithm denoted as R-EA with the simulation experiment on the MATLAB platform. The simulation environment and the initial conditions are set as follows:

(1) Wireless sensor network consists of 50 sensor nodes. The nodes are randomly distributed in a $100 \times 100 \mathrm{~m}$ sensing region;

(2) The sensing radius of the sensor nodes is $250 \mathrm{~m}$ and the communication radius is $200 \mathrm{~m}$;

(3) The 10 interference sources are randomly deployed in the sensing area;

(4) The interference source signal the acceleration is $10 \mathrm{bits} / \mathrm{s}$. The maximum speed is $50 \mathrm{bits} / \mathrm{s}$.

In order to evaluate the effectiveness, the following performance evaluation metrics are given:

(1) Signal strength: received signal strength at the receiving end;

(2) Signal void ratio: the ratio of the signal hole symbols number and the signal symbols number.

Figure 3 shows the signal strength of the two algorithms. HR-DAC algorithm can sense the direction of the omnidirectional, directional, and custom sensor densities according to the sensor sensing direction and deployment 


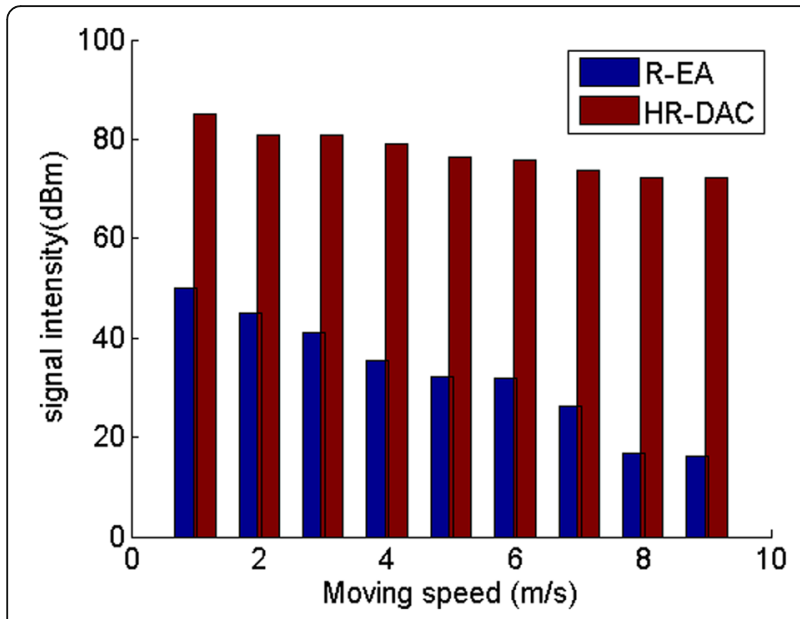

Fig. 3 Signal intensity

requirements. HR-DAC algorithm uses the multi-direction connected graph to analyze the sensor's exposure path and the hidden path problem. HR-DAC algorithm uses multidimensional connectivity graph to analyze the density of sensor networks for continuous jitter problem. HR-DAC algorithm uses the sensor density prediction method to analyze the random deployment of sensor nodes. Therefore, the signal intensity of the HR-DAC algorithm is significantly higher than that of the R-EA algorithm. And HR-DAC algorithm can reduce the impact of high-speed signal transmission on the signal strength.

Figure 4 shows the comparison of the void ratio of the two algorithms. In the diversity of sensor network channel model, the HR-DAC algorithm can detect time domain channel impulse response signal according to the regional geometry and density. HR-DAC algorithm finds the empty signal through the analysis of linear resolution and mobile intelligent network signal detection. HRDAC algorithm can repair the signal holes by traversing

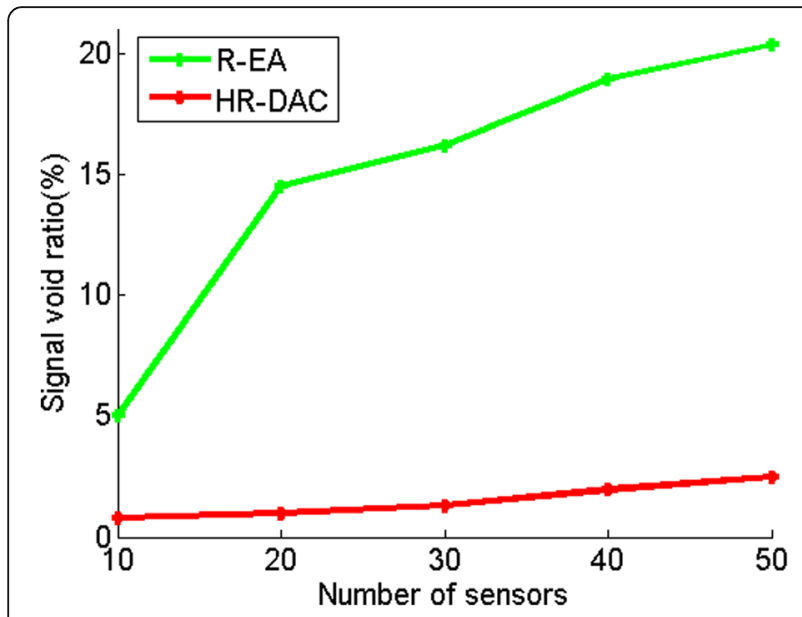

Fig. 4 Signal void ratio the connectivity graph of the mobile crowd network, combined with the crowd signal fusion degree. So, the HRDAC algorithm can significantly reduce signal void ratio. And HR-DAC algorithm can provide signal reliability guarantee for high-density wireless sensor networks.

\section{Conclusions}

With the growth of the network scale and the complex harsh environment, the wireless sensor network has a number of signal holes at the same time. In order to repair the holes, we proposed a sensor deployment density-aware mobile crowd network signal hole repair strategy. Firstly, the density of the sensor network is analyzed by using multi-dimensional connected graph. The sensor nodes are randomly deployed by using the sensor density prediction method. Sensor deployment density sensing model is proposed. Secondly, the diversity of the sensor network channel model can detect time domain channel impulse response signal according to the regional geometry and density detection. Finally, the network signal is detected by the signal detection of the mobile crowd network. Experimental results show that the proposed algorithm can effectively enhance the signal strength and reduce the rate of empty signal.

\section{Competing interests}

The authors declare that they have no competing interests.

Received: 3 August 2016 Accepted: 10 December 2016

Published online: 05 January 2017

\section{References}

1. J Jin, A Sridharan, B Krishnamachari et al., Handling inelastic traffic in wireless sensor networks[J]. IEEE Journal on Selected Areas in Communications 28(7), 1105-1115 (2010)

2. E Lee, S Park, F Yu et al., Communication model and protocol based on multiple static sinks for supporting mobile users in wireless sensor networks[J]. IEEE Transactions on Consumer Electronics 56(3), 1652-1660 (2010)

3. X Han, X Cao, EL Lloyd et al., Fault-tolerant relay node placement in heterogeneous wireless sensor networks[J]. IEEE Transactions on Mobile Computing 9(5), 643-656 (2010)

4. CT Cheng, CK Tse, FCM Lau, A delay-aware data collection network structure for wireless sensor networks[J]. IEEE Sensors Journal 11(3), 699-710 (2011)

5. A Bertrand, J Szurley, P Ruckebusch et al., Efficient calculation of sensor utility and sensor removal in wireless sensor networks for adaptive signal estimation and beamforming[J]. IEEE Transactions on Signal Processing 60(11), 5857-5869 (2012)

6. Cl Chang, Multiparameter receiver operating characteristic analysis for signal detection and classification[J]. IEEE Sensors Journal 10(3), 423-442 (2010)

7. MU Mahfuz, D Makrakis, HT Mouftah, A comprehensive analysis of strength-based optimum signal detection in concentration-encoded molecular communication with spike transmission.[J]. IEEE Transactions on Nanobioscience 14(1), 67-83 (2015)

8. FA Bhatti, GB Rowe, KW Sowerby et al., Blind signal detection using a linear antenna array: an experimental approach[J]. IEEE Transactions on Vehicular Technology 63(3), 1135-1145 (2014)

9. C Lei, J Zhang, Q Gao, Detection of unknown and arbitrary sparse signals against noise[J]. let Signal Processing 8(2), 146-157 (2014) 
10. X Gao, L Dai, Y Hu et al., Low-complexity signal detection for large-scale MIMO in optical wireless communications[]]. IEEE Journal on Selected Areas in Communications 33(9), 1-1 (2015)

11. Naresh M, Venkat Reddy D, Ramalinga Reddy K. Vertical handoff combined with local route repair based on SINR matrix to improve performance of 4G-Multiradio Mesh Network[C]// International Conference on Communications and Signal Processing. 2014;127-131.

12. Cadambe $\vee \mathrm{R}$, Jafar $\mathrm{S} A$, Maleki H. Minimum repair bandwidth for exact regeneration in distributed storage[C]// Wireless Network Coding Conference. 2010;1-6.

\section{Submit your manuscript to a SpringerOpen ${ }^{\circ}$ journal and benefit from:}

- Convenient online submission

- Rigorous peer review

- Immediate publication on acceptance

- Open access: articles freely available online

- High visibility within the field

- Retaining the copyright to your article

Submit your next manuscript at $>$ springeropen.com 